\title{
Update on femoral neck fracture in children: treatment and complications
}

Bernardo WM, Akkari M, Santili C, Akel E, Angelim R

http://dx.doi.org/10.1590/1806-9282.61.01.004

1. Most relevant risk factors for the development of avascular necrosis after femoral neck fractures do NOT include:

a. Type of fracture.

b. Occurrence of deviation.

c. Gender and race.

d. Quality of reduction.

2. Surgical or conservative treatment?

a. Closed reduction is recommended.

b. Fracture surgical fixation is recommended.

c. Immobilization is sufficient.

d. Reduction, immobilization and late surgery.

3. What kind of reduction (open or closed) is most appropriate in this type of fracture?

a. Closed treatment is the rule.

b. Always closed reduction.

c. Always open reduction.

d. Anatomical reduction (closed or open).

4. Does early hip decompression reduce the risk of avascular necrosis (AVN)?

a. Decompression of the early hematoma reduces the risk of AVN.

b. This does not interfere with the rate of AVN.

c. Decompression must be delayed to avoid infection.

d. It depends on the success of the closed reduction.

5. What is the maximum time between the accident and early therapeutic approach to minimize the most common complications?

a. The first 12 hours.

b. The first 24 hours.

c. The first 48 hours.

d. The first 72 hours.
ANSWERS TO CLINICAL SCENARIO: DEGENERATIVE SPONDYLOLISTHESIS: SURGICAL TREATMENT [Published in 2014; 60(6)]

1. Is the use of bone substitutes such as BMP (bone morphogenetic protein) safe and effective in lumbosacral arthrodesis?

Complications include osteolysis and heterotopic ossification. (Alternative $\mathbf{B}$ )

2. Bone substitutes are equal or superior to autografts in this situation?

The association of local bone graft (from the posterior vertebral elements) and beta-tricalcium phosphate is a therapeutic option. (Alternative $\mathbf{C}$ )

3. What is the most appropriate diagnostic study in this clinical context?

The most appropriate diagnostic test in this situation is plain radiography. (Alternative $\mathbf{D}$ )

4. Is it necessary to refer the patient to arthrodesis with use of rigid pedicle screws (non-dynamic)? Instrumented fusion produces less progression and improved walking ability. (Alternative B)

5. For how long should non-surgical treatment be conducted?

After 12 weeks of failure in conservative treatment. (Alternative $\mathbf{C}$ ) 